\title{
Vorbeugen ist besser als heilen!
}

A nlässlich der ersten Lesung des Gesetzesentwurfs zur Stärkung der gesundheitlichen Prävention am 2. Februar 2005 erklärte die Bundesgesundheitsministerin, hiermit eine breite gesellschaftliche Initiative zur Gesundheitsvorsorge starten und Menschen zu gesundheitsbewusstem, Krankheiten vorbeugendem Verhalten ermuntern zu wollen. Damit soll die gesundheitliche Prävention zur eigenständigen Säule im Gesundheitssystem ausgebaut werden. Prävention, Therapie, Rehabilitation und Pflege stehen zukünftig gleichrangig neben einander. Dies bedeutet auch eine Neuorientierung in unserem traditionell kurativ ausgerichteten Gesundheitssystem. Die von der Ministerin beispielhaft erwähnten Erkrankungen Diabetes, Herz-Kreislauf-Krankheiten und Rückenleiden sind bedeutsame Massenphänomene, die sich gut für gezielte und effektive Präventionsmaßnahmen eignen.

Auch allergische Erkrankungen erfüllen in idealtypischer Weise die Voraussetzungen zur primärund sekundärpräventiven Intervention, wie sie im Gesetzestext gefordert werden. Diese betreffen die Verbreitung, die Verteilung auf bestimmte Bevölkerungsgruppen, insbesondere nach Alter, Geschlecht und sozialer Lage, die gesundheitliche und ökonomische Be"Pråvention, Therapie,
Rehabilitation und Pflege stehen künftig gleichrangig nebeneinander. Dies bedeutet eine Neuorientierung in unserem traditionell kurativ ausgerichteten Gesundheitssystem."

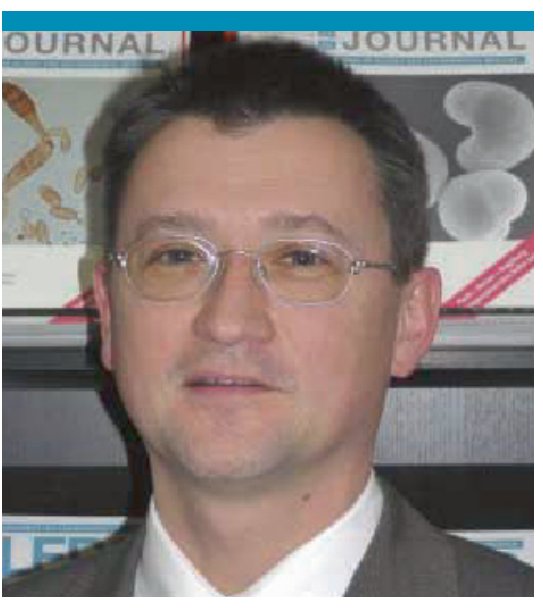
deutung und die Möglichkeiten und den Aufwand der Beeinflussung durch Maßnahmen von primärer Prävention und Gesundheitsförderung.

Insbesondere vor dem Hintergrund der in den letzten Jahrzehnten stark gestiegenen Allergiehäufigkeit und des weitgehenden Fehlens einer kausalen Therapie kommt der Primär- und Sekundärprävention besondere Bedeutung zu. Dies hat auch das Bundesgesundheitsministerium erkannt und vor einigen Jahren das Aktionsbündnis Allergieprävention mitinitiiert und unterstützt. Bedauerlich ist, dass diese Un-
Prof. Dr. Torsten Schäfer, Institut für Sozialmedizin, Universitätsklinikum Schleswig-Holstein, Lübeck

terstützung im Moment ausgesetzt ist. Dies um so mehr, als sich das Aktionsbündnis bei eher bescheidener Finanzierung als höchst effizient erwiesen hat. Auf der Basis breiter und interdisziplinärer Sachkunde wurden mit viel Eigenengagement zahlreiche Projekte zur Allergieprävention initiiert und durchgeführt. Darüber hinaus konnte das Aktionsbündnis Allergieprävention evidenzbasierte und konsentierte Empfehlungen zur Allergieprävention erarbeiten, die von der AWMF als erste S3-Leitlinie der Bereiche Allergologie und Dermatologie an-

Interventionen selbst in Risikopopulationen wie Atopikerfamilien verbesserungsfähig sind. Die Sachverständigen gehen hier von einem erheblichen ungenutzten präventiven Potential aus. Eine Umsetzung des Präventionsgesetzes ohne die Berücksichtigung allergischer Erkrankungen erscheint mir daher nicht sinnvoll.

Die Inhalte der Präventionsempfehlungen haben ihre Evidenzbasis in Studien der Grundlagenforschung wie auch in der epidemiologischen Forschung. Da die genetische Prädisposition bislang nicht beeinflussbar ist, beziehen sich die Empfehlungen auf Umweltfaktoren, die Allergie fördernd oder präventiv wirksam sein können. Die Arbeitsgruppen „Umweltmedizin“ (Sprecher Dr. M. Schümann, Hamburg) und „Epidemiologie allergischer und dermatologischer Erkrankungen" (Sprecher Prof. Dr. T. Schäfer, Lübeck) der Deutschen Arbeitsgemeinschaft für Epidemiologie hielten im November des vergangenen Jahres einen gemeinsamen Workshop zum Thema Allergie und Umwelt in Lübeck ab. Nahezu alle auf diesem Gebiet tätigen Arbeitsgruppen stellten hier ihre neuesten Forschungsergebnisse vor. Acht der insgesamt vierzehn Beiträge finden Sie auf den Seiten 107-124 dieser Ausgabe des Allergo Journal in Kurzform. Ich wünsche Ihnen eine anregende Lektüre.

Vorbeugen ist besser als heilen - und dies gilt insbesondere, wenn heilen - wie bei Allergien - nur schwer möglich ist.

erkannt wurden. Sie stehen als Grundlage für die Entwicklung entsprechender Präventionsprogramme zur Verfügung.

Mit Recht wies schon vor 4 Jahren der Sachverständigenrat für die konzertierte Aktion im Gesundheitswesen in seinem Gutachten zur Über-, Unterund Fehlversorgung am Beispiel des Asthmas darauf hin, dass durch entsprechende Präventionsmaßnahmen der Entwicklung allergischer Erkrankungen vorgebeugt werden kann. Festgestellt wurde aber auch, dass der Kenntnisstand und der Umsetzungsgrad solcher
Es grüßt Sie herzlich,

Ihr

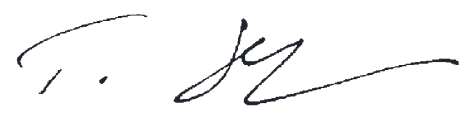

Univ.-Prof. Dr. Torsten Schäfer, MPH 